\title{
Changes in bone resorption and vascular endothelial growth factor after a single zoledronic acid infusion in cancer patients with bone metastases from solid tumours
}

\author{
DANIELE SANTINI ${ }^{1}$, BRUNO VINCENZI $^{1}$, ROSEMARY A. HANNON ${ }^{3}$, JANET E. BROWN ${ }^{4}$, \\ GIORDANO DICUONZO ${ }^{2}$, SILVIA ANGELETTI ${ }^{2}$, ANNALISA LA CESA ${ }^{1}$, ROBERT E. COLEMAN ${ }^{4}$, \\ GIUSEPPE TONINI $^{1}$, ALFREDO BUDILLON ${ }^{5}$, MICHELE CARAGLIA $^{5}$ and INGUNN HOLEN ${ }^{4}$ \\ ${ }^{1}$ Clinical Oncology, ${ }^{2}$ Clinical Pathology, University Campus Bio-Medico, Rome, Italy; \\ ${ }^{3}$ Academic Unit of Bone Metabolism, Division of Clinical Sciences (North), University of Sheffield, \\ Sheffield S5 7AU; ${ }^{4}$ Clinical Oncology, Division of Genomic Medicine, University of Sheffield, \\ Sheffield S10 2RX, UK; ${ }^{5}$ Experimental Pharmacology Unit, Department of Experimental Oncology, \\ National Institute of Tumours 'Fondazione G. Pascale', Naples, Italy
}

Received July 12, 2005; Accepted August 22, 2005

\begin{abstract}
Zoledronic acid (Zometa, ZOL) is increasingly used to treat tumour-induced bone disease, and is also reported to have antiangiogenic properties in vivo. In this study, we have investigated the correlations between changes in the proangiogenic cytokine, vascular endothelial growth factor (VEGF), and markers of bone resorption in a cohort of patients with metastatic bone disease, following a single infusion of ZOL. Twenty-four consecutive selected cancer patients with scintigraphic and radiographic evidence of bone metastases were treated for the first time with a single infusion of $4 \mathrm{mg}$ ZOL. Patients were considered ineligible if they had received any steroid therapy, radiotherapy, chemotherapy, immunotherapy or haemopoietic growth factors in the 4 weeks before or during the study period. Circulating levels of VEGF and $\beta$ crosslinked type I collagen C-telopeptide (BCTX) were measured at base-line and at 1, 2, 7 and 21 days following ZOL infusion. The majority of our patients (23/24) developed a significant reduction in circulating levels of BCTX at just 1 day after the single zoledronic acid infusion, median percentage decrease $67.05 \%$ (95\% CI, 52.39\%; 76.27\%).
\end{abstract}

Correspondence to: Dr Daniele Santini, Clinical Oncology, University Campus Bio-Medico, Via Emilio Longoni 69, 00155 Rome, Italy

E-mail: d.santini@unicampus.it

Dr Michele Caraglia, Experimental Pharmacology Unit, Department of Experimental Oncology, National Cancer Institute 'Fondazione G. Pascale', Via M. Semmola, 80131 Naples, Italy

E-mail: michele.caraglia@fondazionepascale.it

Key words: angiogenesis, bone metastases, marker of bone resorption, zoledronic acid
This reduction persisted at all following time points in almost all subjects in our patient population (day 2, 95.8\%; day 7 , $100 \%$; day $21,91.7 \%$ ). The median decrease at day 2 was $85.67 \%$ (95\% CI, 78.23\%; 90.16\%); at day 7, 67.38\% (95\% CI, 67.38\%; 86.98); and at day 21, 76.89\% (95\% CI, 35.00\%; $83.16 \%$ ). Moreover, a linear regression model with variance analysis demonstrated a statistically significant correlation between median VEGF and BCTX circulating levels at each of the time points (1, 2, 7 and 21 days after ZOL infusion). The present work demonstrates that a single infusion of ZOL was able to induce a rapid and long lasting decrease of BCTX plasma levels in the majority (23/24) of the included cancer patients. Furthermore, we found that there is a correlation between the levels of VEGF and BCTX following ZOL treatment. Future clinical trials should be designed to prospectively evaluate the prognostic role of reduction of BCTX and VEGF in response to ZOL to predict clinical and skeletal outcome.

\section{Introduction}

Bone metastases are common in many advanced cancers and are clinically a considerable source of skeletal morbidity. Bisphosphonates are potent inhibitors of osteoclast activity that have demonstrated efficacy in the treatment of bone metastases $(1,2)$. Bisphosphonates are extensively used for the treatment of metabolic bone disease because they bind avidly to the bone mineral at sites of active bone remodelling, where they achieve therapeutic concentrations (1-3). Bound bisphosphonates are subsequently released during bone resorption and are internalized by osteoclasts, leading to induction of osteoclast apoptosis and inhibition of bone resorption (4-6). Type I collagen is the predominant protein in bone and its breakdown products are being increasingly investigated as markers of bone resorption in metastatic bone disease (7). Recent studies on the effect of ZOL on bone resorption in patients with tumour-induced bone disease have shown significant and rapid decreases in the bone resorption markers, 
crosslinked $\mathrm{N}$ - and C-terminal type I collagen telopeptides, NTX and CTX (8-10). Several urinary and serum markers of bone resorption have recently been demonstrated to be predictive of skeletal complications in prostate cancer, lung cancer and other solid tumours and to be useful for monitoring responses to bisphosphonate therapy (11-13). In cancer patients with lytic bone disease, suppression of NTX correlated with a reduction in fracture risk and a significant reduction in bone disease progression (14). There is now extensive preclinical evidence that bisphosphonates also have antitumour activity, by causing reduced proliferation and viability of tumour cell lines in vitro as well as reduced skeletal tumour burden and slower progression of bone lesions in animal models (15-23). Several mechanisms have been proposed to explain these observations. Bisphosphonates may render the bone a less favorable microenvironment for tumour cell growth by reducing bone resorption and local release of growth factors. Bisphosphonates may also have direct antitumour effects, as they have been shown to inhibit proliferation and induce apoptosis of a variety of human tumour cell lines in vitro (15-23). In addition, bisphosphonates have been reported to inhibit tumour cell adhesion to the extracellular bone matrix and tumour cell invasion, and to have antiangiogenic and immunomodulatory activities. The mechanisms responsible for the observed antitumour effects of bisphosphonates are beginning to be elucidated.

Recent evidence suggests that part of the antitumour activity of bisphosphonates may be attributed to an antiangiogenic effect. Wood et al (24; Proc ASCO 19: abs. 664, 2000) reported that fetal calf serum, basic fibroblast growth factor (bFGF), and vascular endothelial growth factor (VEGF)induced endothelial cell proliferation is inhibited by ZOL in an in vitro model of angiogenesis. Moreover, ZOL was shown to reduce vessel sprouting in cultured aortic rings and in the chicken egg chorioallantoic membrane assay. Finally, Fournier et al, in a subcutaneous testosterone implant model in castrated rats, demonstrated that zoledronic acid treatment strongly inhibits the angiogenic response induced by bFGF and VEGF (25). Our research group was the first to report a significant decrease of circulating levels of VEGF in bone metastatic cancer patients receiving a single, first dose of pamidronate or ZOL $(26,27)$. Collectively, these data suggest that $\mathrm{ZOL}$ and other bisphosphonates may play a clinical role in the reduction of skeletal tumour burden and the prevention of bone metastasis.

The aims of the present study were, firstly, to confirm the early changes in bone resorption in response to a single $\mathrm{ZOL}$ infusion in consecutive patients with bone metastases and, secondly, to investigate the correlations between the modifications of markers of bone resorption and the modifications of circulating levels of VEGF in these patients.

\section{Patients and methods}

Patients. Twenty-four consecutive patients ( 9 males, 15 females), aged 44-76 years (median age, 65), with advanced solid cancer and bone metastases, were included in the study (patients' characteristics are shown in Table I). Patients were considered eligible for the study if they had a histologically confirmed solid cancer associated with scintigraphic
Table I. Patients' characteristics.

\begin{tabular}{lc}
\hline Total $(\%)$ & $24(100)$ \\
Median age in years (range) & $65(44-76)$ \\
Male/female $(\%)$ & $37.5 / 62.5$ \\
Median performance status & \\
ECOG score (range) & $1(0-2)$ \\
$0-1$ score/2 score $(\%)$ & $62.5 / 37.5$ \\
Cancer type $(\%)$ & \\
Breast carcinoma & $14(58.3)$ \\
Prostate adenocarcinoma & $4(16.7)$ \\
Non-small cell lung cancer & $3(12.5)$ \\
Other primary cancers & $3(12.5)$
\end{tabular}

Bone segments involved

by metastases (range)

Previous chemotherapy (\%)

Concurrent hormonal therapy (\%)

Metastases other than bone

locations (patients) $(\%)$

No other locations

Lung metastases

Liver metastases

Lung + liver metastases

Other locations

identification and radiographic confirmation of bone metastases. In addition, patients were required to have, at study entry, a neutrophil count $\geq 1.5 \times 10^{9} / 1$, a platelet count $>100 \times 10^{9} / 1$, normal hepatic and renal function and no acute or chronic infections or inflammatory diseases. Patients were considered ineligible if they had reported fever (body temperature $>38.0^{\circ} \mathrm{C}$ ) in the last week before study entry or had received any radiotherapy, chemotherapy, immunotherapy or haemopoietic growth factors in the 4 weeks before entry into the study. Patients who recently $(<1$ week) or simultaneously received steroid treatment were considered ineligible for the study. Patients previously treated with any bisphosphonates were excluded from the study. Hormonal therapy was allowed only if it had started at least 3 months prior to accrual. All patients received ZOL on an outpatients basis. All patients were $>18$ years of age and had given their informed consent according to local guidelines with the approval of the appropriate ethics committee.

Treatment and follow-up investigation. All patients received $4 \mathrm{mg}$ of ZOL (Zometa ${ }^{\circledR}$, Novartis) in $100 \mathrm{ml}$ of $0.9 \%$ saline over a period of $15 \mathrm{~min}$ as an intravenous infusion starting at 10:00 a.m. Venous blood for VEGF and BCTX assessment was drawn into an EDTA anticoagulant tube before 10:00 a.m. after an overnight fast at baseline, just before the beginning of drug infusion, and again at 1, 2, 7 and 21 days after the ZOL infusion. After drawing, the venous blood sample was 


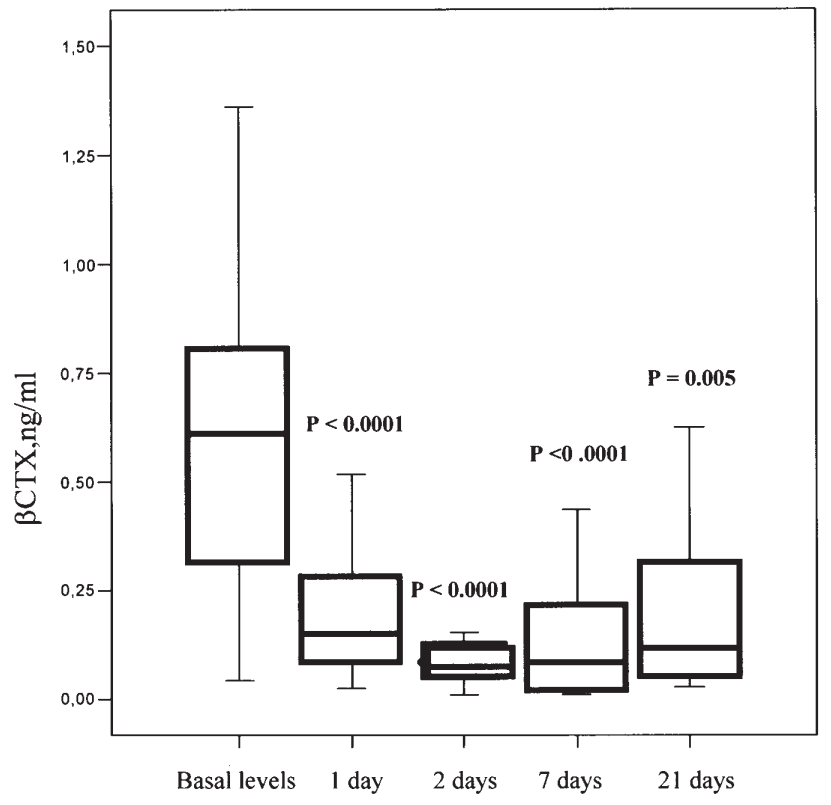

Time points

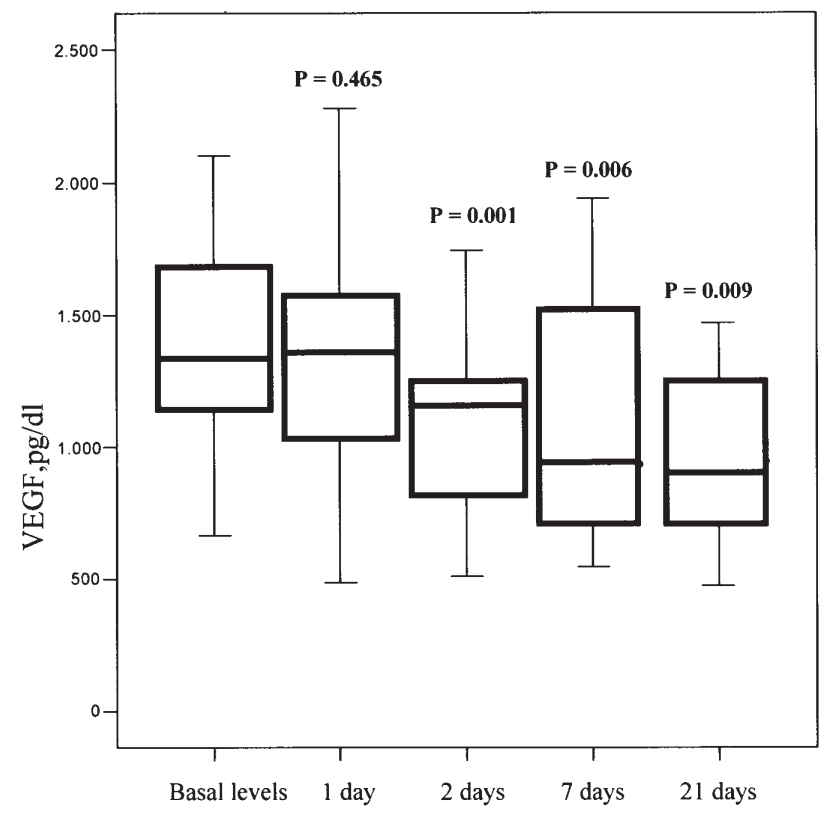

Time points

Figure 1. BCTX and VEGF levels at days 1,2, 7 and 21 after ZOL administration. Red boxes represent 95 percentiles. Horizontal black bars in the red boxes represent the median value. Bottom and top horizontal bars indicate minimum and maximum values. P-values are calculated according to Wilcoxon's test for non-parametric dependent continuous variable.

Table II. BCTX and VEGF circulating level modifications after a single infusion of zoledronic acid.

\begin{tabular}{lccc}
\hline BCTX & $\begin{array}{c}\text { Median value } \\
(\mathrm{ng} / \mathrm{ml})\end{array}$ & $\begin{array}{c}95 \% \text { confidence } \\
\text { interval }(\mathrm{ng} / \mathrm{ml})\end{array}$ & P-value \\
\hline Basal levels & 0.6417 & $0.408-1.010$ & - \\
1 day & 0.216 & $0.122-0.455$ & $<0.0001$ \\
2 days & 0.104 & $0.066-0.162$ & $<0.0001$ \\
7 days & 0.127 & $0.105-0.272$ & $<0.0001$ \\
21 days & 0.200 & $0.145-0.366$ & 0.005 \\
& & & \\
\hline & & $95 \%$ confidence & P-value \\
VEGF & Median value & interval (pg/dl) & \\
\hline Basal levels & 1371.500 & $1200.240-1681.764$ & - \\
1 day & 1368.000 & $1081.336-1540.442$ & $0.390^{a}$ \\
2 days & 1065.000 & $854.967-1220.589$ & 0.005 \\
7 days & 965.500 & $907.717-1346.173$ & 0.016 \\
21 days & 960.500 & $814.291-1275.578$ & 0.006 \\
\hline
\end{tabular}

${ }^{\mathrm{a} N o t}$ significant.

rapidly centrifuged for $10 \mathrm{~min}$ at $10,000 \mathrm{rpm}$ and plasma stored at $-80^{\circ} \mathrm{C}$ until tested for VEGF and BCTX levels. White blood cell and platelet counts, haemoglobin levels and serum total calcium levels were also determined at the same time points.

$V E G F$ and $\beta C T X$ analysis. VEGF was assayed using the R\&D quantitative kit according to the manufacturer's instructions
(R\&D Systems, Minneapolis, MN). The detection limit of the VEGF was $62.5 \mathrm{pg} / \mathrm{ml}$.

Plasma BCTX was assayed by electrochemiluminescent assay on an Elecsys 2010 auto analyser (Roche Diagnostics $\mathrm{GmBH}$, Penzberg, Germany). The detection limit of the assay was $0.01 \mathrm{ng} / \mathrm{ml}$. The interassay $\mathrm{CV}$ was $6.5 \%$ at $0.7 \mathrm{ng} / \mathrm{ml}$. All samples were centrifuged at $1200 \times \mathrm{g}$ for $5 \mathrm{~min}$ before analysis. In both assays, all samples from the same individual were analysed in the same analytical batch.

Statistical analysis. Basal VEGF and BCTX levels were compared to the values observed at 1,2, 7 and 21 days after ZOL infusion using the Wilcoxon's test for non-parametric dependent continuous variables. A linear regression model with variance analysis was used to correlate VEGF and BCTX circulating levels at the different time points. At the last time point, 6 samples were missing; no correction was made for missing data. A two-tailed P-value was considered significant when $<0.05$. SPSS software (version 11.5, SPSS, Chicago, IL) was used for statistical analysis.

\section{Results}

$\beta C T X$ analysis. Table II and Fig. 1 show the median circulating levels after a single infusion of zoledronic acid. The median BCTX basal level was $0.6417 \mathrm{pg} / \mathrm{dl}$ (95\% CI, 0.408-1.010). These levels significantly decreased 1 day after ZOL infusion to $0.216 \mathrm{ng} / \mathrm{ml}(95 \% \mathrm{CI}, 0.122-0.455)(\mathrm{P}<0.0001)$. This effect persisted throughout the 21-day follow-up period.

In the majority of patients (23/24), circulating levels of BCTX were significantly reduced 1 day after the single zoledronic acid infusion, median decrease $67.05 \%$ (95\% CI, 52.39\%; $76.27 \%$ ). This reduction was found at all of the time points 
Table III. Median percentage changes in circulating BCTX and VEGF levels after zoledronic acid infusion at different time points.

\begin{tabular}{lcc}
\hline & Median reduction $(\%)$ & $95 \%$ CI $(\%)$ \\
\hline BCTX & & \\
1 day & -67.05 & $-52.39 ;-76.27$ \\
2 days & -85.67 & $-78.23 ;-90.16$ \\
7 days & -67.38 & $-67.38 ;-86.98$ \\
21 days & -76.89 & $-35.00 ;-83.16$ \\
VEGF & & \\
1 day & -6.71 & $+8.70 ;-16.08$ \\
2 days & -23.82 & $+1.47 ;-30.88$ \\
7 days & -27.75 & $-2.03 ;-34.69$ \\
21 days & -39.42 & $-15.09 ;-44.13$ \\
\hline
\end{tabular}

in almost all our patients (day 2, 95.8\%; day 7,100\%; day $21,91.7 \%)$. The median percentage changes in circulating ßCTX levels after ZOL infusion at different time points are summarized in Table III.

VEGF analysis. The median VEGF basal value was not significantly reduced 1 day after the single infusion of $\mathrm{ZOL}$ (1371.50 pg/dl; 95\% CI, 1200.24-1681.76) vs $1368.00 \mathrm{pg} / \mathrm{dl}$; 95\% CI, 1081.33-1540.44) $(\mathrm{P}=0.390)$. However, 2 days after ZOL infusion, VEGF levels showed a statistically significant decrease compared to the basal levels (1065.00 pg/dl; 95\% CI, 854.96-1220.58) $(\mathrm{P}=0.005)$. This effect on circulating VEGF levels persisted throughout the study. These results are summarized in Table II and Fig. 1. The median reductions in percentage (\%) of circulating VEGF levels after ZOL infusion at different time points are summarized in Table III.

Correlation between $\beta C T X$ and VEGF circulating levels. A linear regression model with variance analysis failed to show a significant positive correlation between basal VEGF and $B C T X$ values $(B$ regression coefficient $=0.322 ; \mathrm{P}=0.125$ ) (Table IV). In contrast, a statistically significant correlation between median VEGF and BCTX circulating levels was found at each of the time points. In particular, a strong direct correlation was identified just 1 day after the ZOL single infusion ( $B$ regression coefficient $=0.627 ; \mathrm{P}=0.002$ ) which persisted for 2 days ( $\beta$ regression coefficient $=0.655 ; P=0.001$ ) and reached the highest level of significance at 7 days after infusion $(B$ regression coefficient $=0.872 ; \mathrm{P}<0.0001$ ). Furthermore, the correlation persisted for 21 days after the infusion ( $\beta$ regression coefficient $=0.511 ; \mathrm{P}=0.018$ ). These results are summarized in Table IV and Fig. 2.

Therefore, we arbitrarily decided to divide the patients into 'responders' and 'non-responders' on the basis of BCTX and VEGF concomitant decrease at 7-day and 21-day time points. We defined 'responder' patients as those who concomitantly showed a VEGF reduction $>25 \%$ of the basal levels (28) and a BCTX reduction $>50 \%$ of the basal values $(29,30) 7$ or 21 days after the first ZOL infusion. According to these definitions, $45.8 \%$ of patients (11 patients) were
Table IV. Correlation between BCTX and VEGF circulating levels at different time points.

\begin{tabular}{lcc}
\hline Time points & $\beta$ regression coefficient & P-value \\
\hline Basal & 0.322 & $0.125^{\mathrm{a}}$ \\
1 day & 0.627 & 0.002 \\
2 days & 0.655 & 0.001 \\
7 days & 0.872 & $<0.0001$ \\
21 days & 0.511 & 0.018 \\
\hline
\end{tabular}

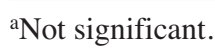

'responders' at the 7-day time point and $58.3 \%$ (14 patients) were 'responders' at the 21-day time point. We did not demonstrate any correlation between basal levels of BCTX and response to ZOL (data not shown).

Secondary parameters. No significant differences in platelet level, white blood cell count, haemoglobin concentration and renal function were recorded before and 1, 2, 7 and 21 days after ZOL infusion. However, as expected, a statistically significant decrease in serum total calcium levels was observed after bisphosphonate administration: the median calcium level before ZOL administration was $11.31 \mathrm{mg} / \mathrm{dl}$ (range: 9.08-13.1 $\mathrm{mg} / \mathrm{dl})$, while the median value 7 days after was $8.38 \mathrm{mg} / \mathrm{dl}$ (range: 7.2-10.8 mg/dl) $(\mathrm{P}=0.0002)$. Moreover, 4 of 24 patients showed hypocalcemia 21 days after the infusion of ZOL and a second administration had to be changed from 1 to 3 weeks. The changes in serum total calcium did not correlate with the observed changes in circulating VEGF levels following ZOL infusion at any time point. As expected, a significant correlation was identified between serum total calcium concentration and BCTX level 7 days $(B$ regression coefficient $=4.980 ; \mathrm{P}=0.009)$ and 21 days $(\beta$ regression coefficient $=5.521 ; \mathrm{P}=0.006$ ) after $\mathrm{ZOL}$ infusion. Only a borderline statistical significance was noted between BCTX and calcium levels at the basal time ( regression coefficient $=3.357 ; \mathrm{P}=0.061$ ).

A significant correlation in a linear regression model was noted between basal VEGF level and basal platelet count $(ß$ regression coefficient $=4.653 ; \mathrm{P}=0.014)$.

\section{Discussion}

Tumour cells release a variety of growth factors that promote bone resorption and increase the risk of skeletal complications. Recently, bone marker levels have been demonstrated to be representative of neoplastic osteolysis and predictive of negative clinical outcomes in patients with bone metastases secondary to prostate cancer and to NSCLC and other solid tumours. In particular, urinary N-telopeptide level was shown to be a consistent prognostic indicator for all tumour types, reflecting the key role of osteolysis in the development of skeletal complications $(7,11)$. Bisphosphonates are potent inhibitors of osteoclast activity that have demonstrated efficacy in the treatment of tumour-induced bone disease. Bisphosphonates bind avidly to the bone matrix, are released during 

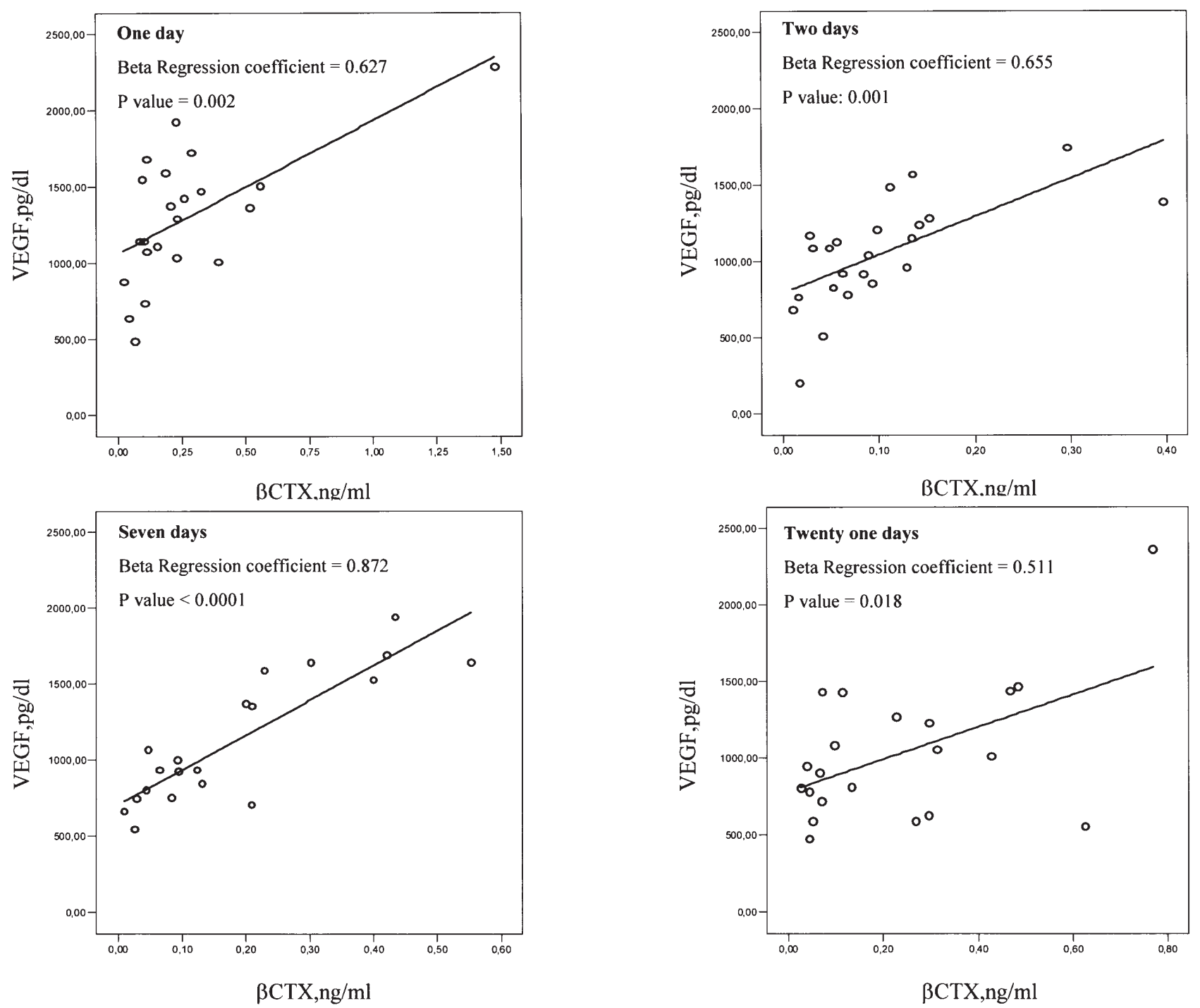

Figure 2. Correlations between median VEGF and BCTX levels 1, 2, 7 and 21 days after ZOL administration. A statistically significant correlation between median VEGF and BCTX circulating levels was found at each of the evaluated time points.

bone resorption, and are subsequently internalized by osteoclasts, where they interfere with biochemical pathways and induce osteoclast apoptosis. Bisphosphonates also antagonize osteoclastogenesis and promote the differentiation of osteoblasts. As a result, bisphosphonates inhibit tumour-induced osteolysis and reduce skeletal morbidity. Garnero et al (31) analysed the changes in serum markers of bone resorption during ZOL therapy in a cohort of patients with Paget's disease. In detail, the urinary excretion of non-isomerized type I collagen C-telopeptide $(\alpha \mathrm{CTX}-\mathrm{I})$ decreased within 5 days with a maximal decrease of $51 \%$ at day $10(\mathrm{P}<0.001$ vs. baseline and placebo), and levels remained suppressed during the 2 months of the study. Moreover, Chen et al (8) analysed the effects of ZOL on markers of bone disease in a sample of cancer patients without any selection regarding the concomitant antineoplastic therapies or the concomitant comorbidities. In this study (8), ZOL produced significant declines from baseline in serum and/or creatinine-corrected urine C-telopeptide, $\mathrm{N}$-telopeptide, pyridinium cross-links, and calcium. The current study is the first to report the early modifications of the levels of markers of bone resorption induced by a single infusion of ZOL in a well characterised cohort of metastatic cancer patients. The present work demonstrates that a single infusion of ZOL is able to induce a rapid and long lasting decrease of BCTX plasma levels in the majority of the patients studied. The significant reduction of BCTX plasma levels is a clear reflection of the inhibition of bone resorption induced by $\mathrm{ZOL}$, including that at sites of bone metastasis. It has been demonstrated that ZOL administered by intravenous infusion ( $4 \mathrm{mg}$ over $15 \mathrm{~min}$ ), results in an abrupt increase of its concentration in peripheral blood, as shown by estimations of the early distribution and elimination of the drug, which are resulting in population half-lives of the drug of about $15 \mathrm{~min}(\mathrm{t} 1 / 2 \alpha)$ and of $1.75 \mathrm{~h}$ $(\mathrm{t} 1 / 2 \beta)$, respectively. Moreover, approximately $55 \%$ of the initial administered dose of the drug is retained in the skeleton and is slowly released back in circulation, resulting in a terminal elimination half-life $(\mathrm{t} 1 / 2 \gamma)$ of about 7 days in population studies $(8,32)$. It has also been reported that a 15-min pulse with ZOL induces either apoptosis or growth 
inhibition in both human prostate and pancreas adenocarcinoma cell lines, starting with osteoclasts after 48-72 h $(23,33)$. Tumour cells secrete themselves or stimulate the production of VEGF by the expression of other growth factors such as TGF $\alpha$ or EGF $(34,35)$. Furthermore, cancer cells can produce bone resorption factors, such as M-CSF or rank ligand, which favour collagen destruction $(36,37)$. Therefore, it is conceivable that reduction of both VEGF and $ß \mathrm{CTX}$ could be recorded from 2 days after ZOL administration. Both the prolonged $\mathrm{ZOL}$ release from the bone and the long recovery from cell death induced by ZOL on both tumour cells and osteoclasts could be the cause of the sustained effects of the lowering of VEGF and CTX plasma levels.

Reports from in vitro systems and animal models have demonstrated that ZOL has antiangiogenic effects. Moreover, as previously described, growing evidence has accumulated in the past years supporting the anticancer effect of ZOL in humans. The in vivo antiangiogenic effects of $\mathrm{ZOL}$ have also been established by the reduction of circulating levels of proangiogenic cytokines in humans induced by a single infusion of the drug $(26,27)$. Recently, a molecular correlate between molecular target of ZOL actions and angiogenesis has been described. In fact, ZOL can induce apoptosis in cancer cells by inactivation of ras (38). The latter is, in turn, involved in the regulation of secretion of angiogenic factors, such as interleukin-8, by both tumour and endothelial cells (39). On the basis of this evidence, we designed this study to evaluate if a correlation between the reduction of VEGF and BCTX circulating levels, a marker of bone resorption, may exist. The present study clearly demonstrates that a statistically significant correlation exists between circulating levels of VEGF and BCTX concentration 1 day after a single infusion of ZOL. This correlation is lasting and persists for 21 days after the infusion. The molecular mechanisms of these correlated events are not known. It is proposed that a "vicious cycle' is created whereby metastatic tumour stimulates bone turnover and bone turnover promotes local tumour growth (40). ZOL has been shown to inhibit the bone turnover, antagonizing the osteoclastogenesis and promoting the differentiation of osteoblasts. As a consequence, the zoledronicinduced inhibition of bone turnover may lead to inhibition of tumour growth in the bone environment; but it is proposed that the tumour growth inhibition induced by $\mathrm{ZOL}$ is in part dependent on angiogenesis inhibition. The changes in BCTX and VEGF in this study may reflect this dual effect of ZOL on tumour growth. In accordance with these results, we identified the percent of decrease of VEGF and BCTX circulating levels in each patient and at each time point and we observed two types of behaviour. We identified a group of patients with a concomitant significant decrease in VEGF and BCTX plasma levels as 'responders' and a second group, in which the decreases in VEGF and BCTX serum levels were not concomitant, as 'non-responders'. This study emphasizes that the biological response to ZOL is not the same in all patients, although the majority had reduced BCTX following a single infusion of the drug. Whereas the molecular mechanisms underlying the reduction in bone resorption markers (inhibition of osteoclast activity) are well established, it is unclear by which mechanisms ZOL causes reduced levels of circulating VEGF, and further studies are required to establish this. The biological and clinical significance of this differential response to $\mathrm{ZOL}$ is not clear, and the number of patients included in this study was not large enough to correlate these changes in BCTX and VEGF with the clinical and skeletal outcome of the disease. Future clinical trials should be designed to prospectively evaluate the role of changes in BCTX and VEGF, separately and in combination, in predicting the clinical and skeletal outcome of ZOL treatment in patients with tumourinduced bone disease.

\section{Acknowledgements}

We thank Dr Monica Marra for helpful discussion of the text and for her technical assistance.

\section{References}

1. Fleisch H: Development of bisphosphonates. Breast Cancer Res 4: 30-34, 2002.

2. Russell RG, Croucher PI and Rogers MJ: Bisphosphonates: pharmacology, mechanisms of action and clinical uses. Osteoporos Int 9: 66-80, 1999.

3. Stronski SA, Bettschen-Camin L, Wetterwald A, et al: Bisphosphonates inhibit 1,25-dihydroxyvitamin D3-induced increase of osteocalcin in plasma of rats in vivo and in culture medium of rat calvaria in vitro. Calcif Tissue Int 42: 248-254, 1988.

4. Flanagan AM and Chambers TJ: Inhibition of bone resorption by bisphosphonates: interactions between bisphosphonates, osteoclasts, and bone. Calcif Tissue Int 49: 407-415, 1991.

5. Rogers MJ, Gordon S, Benford HL, et al: Cellular and molecular mechanisms of action of bisphosphonates. Cancer 88: 2961-2978, 2000.

6. Sato M, Grasser W, Endo N, et al: Bisphosphonate action. Alendronate localization in rat bone and effects on osteoclast ultrastructure. J Clin Invest 88: 2095-2105, 1991.

7. Brown JE, Thompson CS, Ellis SP, et al: Bone resorption predicts for skeletal complications in metastatic bone disease. Br J Cancer 89: 2031-2037, 2003.

8. Chen T, Berenson J, Vescio R, et al: Pharmacokinetics and pharmacodynamics of zoledronic acid in cancer patients with bone metastases. J Clin Pharmacol 42: 1228-1236, 2002.

9. Skerjanec A, Berenson J, Hsu C, et al: Pharmacokinetics and pharmacodynamics of zoledronic acid in cancer patients with varying degrees of renal failure. J Clin Pharmacol 43: 154-162, 2003.

10. Garnero P, Ferreras M, Karsdal MA, et al: The type I collagen fragments ICPT and CTX reveal distinct enzymatic pathways of collagen degradation. J Bone Miner Res 18: 859-867, 2003.

11. Brown JE, Cook RJ, Major P, et al: Bone turnover markers as predictors of skeletal complications in prostate cancer, lung cancer, and other solid tumors. J Natl Cancer Inst 97: 59-69, 2005.

12. Coleman RE, Major P, Lipton A, et al: The predictive value of bone resorption and formation markers in cancer patients with bone metastases receiving the bisphosphonate zoledronic acid. J Clin Oncol (In press).

13. Vinholes J, Guo CY, Purohit OP, et al: Evaluation of new bone resorption markers in a randomized comparison of pamidronate or clodronate for hypercalcemia of malignancy. J Clin Oncol 15: 131-138, 1997.

14. Derenne S, Amiot M, Barille S, et al: Zoledronate is a potent inhibitor of myeloma cell growth and secretion of IL-6 and MMP-1 by the tumoral environment. J Bone Miner Res 14: 2048-2056, 1999.

15. Hiraga T, Williams PJ, Mundy GR, et al: The bisphosphonate ibandronate promotes apoptosis in MDA-MB-231 human breast cancer cells in bone metastases. Cancer Res 61: 4418-4424, 2001.

16. Lee MV, Fong EM, Singer FR, et al: Bisphosphonate treatment inhibits the growth of prostate cancer cells. Cancer Res 61: 2602-2608, 2001.

17. Mackie PS, Fisher JL, Zhou H, et al: Bisphosphonates regulate cell growth and gene expression in the UMR 106-01 clonal rat osteosarcoma cell line. Br J Cancer 84: 951-958, 2001. 
18. Senaratne SG, Pirianov G, Mansi JL, et al: Bisphosphonates induce apoptosis in human breast cancer cell lines. Br J Cancer 82: $1459-1468,2000$

19. Shipman CM, Rogers MJ, Apperley JF, et al: Bisphosphonates induce apoptosis in human myeloma cell lines: a novel antitumour activity. Br J Haematol 98: 665-672, 1997.

20. Shipman CM, Croucher PI, Russell RG, et al: The bisphosphonate incadronate (YM175) causes apoptosis of human myeloma cells in vitro by inhibiting the mevalonate pathway. Cancer Res 58: 5294-5297, 1998.

21. Sonnemann J, Eckervogt V, Truckenbrod B, et al: The bisphosphonate pamidronate is a potent inhibitor of human osteosarcoma cell growth in vitro. Anticancer Drugs 12: 459-465, 2001

22. Takahashi R, Shimazaki C, Inaba T, et al: A newly developed bisphosphonate, YM529, is a potent apoptosis inducer of human myeloma cells. Leuk Res 25: 77-83, 2001.

23. Tassone $\mathrm{P}$, Tagliaferri $\mathrm{P}$, Viscomi C, et al: Zoledronic acid induces antiproliferative and apoptotic effects in human pancreatic cancer cells in vitro. Br J Cancer 88: 1971-1978, 2003.

24. Wood J, Bonjean K, Ruetz S, et al: Novel antiangiogenic effects of the bisphosphonate compound zoledronic acid. J Pharmacol Exp Ther 302: 1055-1061, 2002.

25. Fournier P, Boissier S, Filleur S, et al: Bisphosphonates inhibit angiogenesis in vitro and testosterone-stimulated vascular regrowth in the ventral prostate in castrated rats. Cancer Res 62: 6538-6544, 2002

26. Santini D, Vincenzi B, Avvisati G, et al: Pamidronate induces modifications of circulating angiogenic factors in cancer patients. Clin Cancer Res 8: 1080-1084, 2002.

27. Santini D, Vincenzi B, Dicuonzo G, et al: Zoledronic acid induces significant and long-lasting modifications of circulating angiogenic factors in cancer patients. Clin Cancer Res 9: 2893-2897, 2003.

28. Vincenzi B, Santini D, Dicuonzo G, et al: Zoledronic acidrelated angiogenesis modifications and survival in advanced breast cancer patients. J Interferon Cytokine Res 25: 144-151, 2005.

29. Vinholes JJ, Purohit, OP, Abbey ME, et al: Relationships between biochemical and symptomatic response in a double blind randomised trial of pamidronate for metastatic bone disease. Ann Oncol 8: 1243-1250, 1997.
30. Menssen HD, Sakalova A, Fontana A, et al: Effects of long term intravenous ibandronate therapy on skeletal-related events, survival, and bone resorption markers in patients with advanced multiple myeloma. J Clin Oncol 20: 2353-2359, 2002.

31. Garnero P, Christgau S and Delmas PD: The bisphosphonate zoledronate decreases type II collagen breakdown in patients with Paget's disease of bone. Bone 28: 461-464, 2001.

32. Zhou H, Ma P, Ravera C, Gauron S, et al: Population pharmacokinetics (PK) of Zometa - a third generation bisphosphonate. J Clin Pharmacol 40: 1045-1050, 2000.

33. Tenta R, Tiblalexi D, Sotiriou E, et al: Bone microenvironmentrelated growth factors modulate differentially the anticancer actions of zoledronic acid and doxorubicin on PC-3 prostate cancer cells. Prostate 59: 120-131, 2004.

34. Lee YM, Bae MH, Lee OH, et al: Synergistic induction of in vivo angiogenesis by the combination of insulin-like growth factor-II and epidermal growth factor. Oncol Rep 12: 843-848, 2004.

35. Ongusaha PP, Kwak JC, Zwible AJ, et al: HB-EGF is a potent inducer of tumor growth and angiogenesis. Cancer Res 64 5283-5290, 2004

36. Mundy GR and Yoneda T: Bisphosphonates as anticancer drugs. N Engl J Med 339: 398-400, 1998.

37. Papadopoulos SE, Hamdy NAT and van der Pluijm G: Bisphosphonates in the management of prostatic carcinoma metastatic to the skeleton. Cancer 88: 3047-3053, 2000.

38. Caraglia M, D'Alessandro AM, Marra M, et al: The farnesyl transferase inhibitor R115777 (Zarnestra ${ }^{\circledR}$ ) synergistically enhances growth inhibition and apoptosis induced on epidermoid cancer cells by Zoledronic acid (Zometa ${ }^{\circledR}$ ) and Pamidronate. Oncogene 23: 6900-6913, 2004.

39. Sparmann A and Bar-Sagi D: Ras-induced interleukin-8 expression plays a critical role in tumor growth and angiogenesis. Cancer Cell 6: 447-458, 2004

40. Reddi AH, Roodman D, Freeman C, et al: Mechanisms of tumor metastasis to the bone: challenges and opportunities. J Bone Miner Res 18: 190-194, 2003. 